Original article

\title{
Corticosteroid actions on dengue immune pathology; A review article
}

\author{
S.M. Rathnasiri Bandara ${ }^{\mathrm{a}}$, H.M.M.T.B. Herath ${ }^{\mathrm{b}, *}$ \\ ${ }^{a}$ Kandy general hospital, Sri Lanka \\ ${ }^{\mathrm{b}}$ National hospital of Colombo, Sri Lanka
}

A R T I C L E I N F O

\section{Keywords:}

Dengue

Dengue shock

Corticosteroids

Vasculopathy

Thrombocytopenia

Immune pathology

Review article

\begin{abstract}
A B S T R A C T
Introduction: Dengue infection causes significant morbidity and mortality in over 125 countries worldwide, and its incidence is on the rise. Currently, no therapy is available beyond supportive care. In fact, corticosteroids are used therapeutically for a broad spectrum of diseases including autoimmune, allergic, inflammatory diseases and organ transplant rejection. However, only a few studies were done to evaluate the effectiveness of corticosteroids in dengue infection, although the immune pathology of dengue is similar to other diseases treated effectively by corticosteroids for several decades.

Objectives: This review is aimed at identifying biological actions of steroids at molecular and receptor level in dengue immune pathology after reviewing pharmacological and immunological research findings of corticosteroids and dengue.

Methods: We searched medline/pubmed and Google scholar for publications with the search terms 'dengue' and 'steroid', 'corticosteroid', 'prednisolone', 'methylprednisolone' or 'dexamethasone' in the title and abstract. Then the publications were analyzed according the action of steroids in dengue pathology under different subheadings.

Results: The results are presented under four categories and it shows that corticosteroids can suppress cells involved in innate immunity, T cells, B cells and antibodies,complements and heamatological manifestations in dengue pathology.

Conclusion: This article explains strong supportive evidence for actions of corticosteroids in dengue pathology at receptors and molecular levels. Therefore it is suggested that a gold standard steroid protocol for each phase of dengue pathology that can be tested further with a double blind control trial study.
\end{abstract}

\section{Introduction}

Dengue fever (DF) often presents with fever, rash, headache and myalgia, which are caused by a flavi virus with four distinct stereotypes; DENV-1, DENV-2, DENV-3, and DENV-4. ${ }^{1}$ An infection with one stereotype does not protect against the others and sequential infections put patients at a greater risk for dengue hemorrhagic fever (DHF) and dengue shock syndrome (DSS) ${ }^{2}$ that are mainly caused by immunological dysfunction. ${ }^{3}$ The World Health Organization (WHO) estimates that 50 to 100 million-dengue infections occur annually and it affects more than 125 countries that are known to be dengue endemic. Moreover, it includes 500,000 cases of DHF and 22,000 deaths annually. Most of them are children. The incidence has increased by 30 folds during the past 50 years. ${ }^{4}$ In Sri Lanka, 110,372 cases and 301 deaths were reported within the first seven months of the 2017 epidemic. ${ }^{5}$ Currently, no specific therapy is available beyond supportive care while untreated complicated dengue fever can have a $50 \%$ mortality rate. ${ }^{6}$ Therefore, unless exact or partial treatment is introduced to suppress this life threatening immune dysfunction pathology of dengue, 5 million cases of DHF and 0.22 million deaths will occur within the next decade (2018-2028).

Corticosteroids (CSs) are used therapeutically for a broad spectrum of diseases including autoimmune diseases, allergic and inflammatory diseases and in organ transplant. However, only a limited number of studies were done to evaluate the effectiveness of CSs in dengue infection, ${ }^{7}$ although the immune pathology of dengue has been within the range of immune pathology of other diseases, which have been treated effectively by CSs for several decades.

In fact, fear of administration of corticosteroid to dengue patients still exists because it is an infective illness. Very recently a review article stated favorable effect of steroid in dengue. ${ }^{8}$ In clinical trials there were no evidence of viremia with use of steroids and there were no significant side effects after the administration of low and high oral doses of corticosteroids and high doses of IV corticosteroids. ${ }^{910}$

\footnotetext{
* Corresponding author.

E-mail addresses: rathnasirib68@gmail.com (S.M.R. Bandara), tharukaherath11@gmail.com (H.M.M.T.B. Herath).
} 
Moreover, no evidence of harmful effects of corticosteroids was found ${ }^{11}$ and beneficial effects were found. ${ }^{12}$ In this recent review of dengue and steroids, it was concluded that the effectiveness of corticosteroid for immune suppression in dengue is depended on sustained maintained therapeutic blood levels of corticosteroids using a higher receptor affinity steroids for required time duration.

With the conclusion of clinical trial studies data, a hypothesis was formulated. It is suggested that different doses, roots of administration, and particular groups of steroids contribute to suppress immune pathology of dengue at different stages of dengue infection due to different pharmacological actions of CSs at molecular and receptor levels. This could open a new direction to assess molecular and receptor level evidence of CSs to prove this hypothesis.

\section{Objectives}

This review is aimed at identifying biological actions of steroids at molecular and receptor level in dengue immune pathology after reviewing pharmacological and immunological research findings of CSs and dengue under different subheadings.

\section{Methods}

We searched medline/pubmed and Google scholar for publications with the search terms 'dengue' and 'steroid', 'corticosteroid', 'prednisolone', 'methylprednisolone' or 'dexamethasone' in the title and abstract. Then the publications were analyzed according the action of steroids in dengue pathology under different subheadings.

\section{Results}

The results are presented under four categories depending on steroids action on innate immunity, T cells, B cells and antibodies, complement system and hematological manifestations.

\subsection{CSs suppress cells involved in innate immunity in dengue pathology (Fig. 1)}

Dengue virus (DENV) is presumably injected into the bloodstream during the feeding of mosquitoes on humans and attacks the immune system cells. Firstly, it results in the infection of immature epidermal dendritic cells (DCs) in the epidermis and dermis. ${ }^{13}$ Infected DCs then migrate from the site of infection to lymph nodes where monocytes and macrophages are recruited. Thereafter the infection is amplified and the virus is disseminated through the lymphatic system. ${ }^{14}$ As a result of this primary viremia, several cells of the mononuclear lineage, including blood-derived monocytes, myeloid DCs and splenic and liver macrophages are infected. ${ }^{15}$ The time gap between the bite of an infected mosquito and the onset of clinical symptoms is believed to be 3-15 days. $^{16}$

Immature dendritic cells (imDCs) express high levels of DC-SIGN (Dendritic Cell Specific Intercellular adhesion molecule-3 (ICAM-3) Grabbing Non-integrin)/CD209 which facilitates initial viral binding and entry. CD209 is a known target of dengue virus, having a high affinity for ICAM3 molecules expressed on T-cells. ${ }^{17,18}$ Thus DCs are the most effective antigen presenting cells (APC) able to acquire and display viral antigens and finally activate T-cells. In dengue pathology, infected imDCs also contribute to vascular leak by producing matrix metalloproteinase (MMPs). ${ }^{19}$ The maturation and sensitivity of DCs are promoted by dengue virus induced cytokines e.g. TNF $\alpha$, IFN $\gamma$, IL-6, and activated T cells. ${ }^{3,20}$ Mature Dendritic Cells (mDCs) lose their ability to capture and process antigens, up-regulate their production of cytokines, increase their expression of MHC class which has been suggested as a mechanism by which the immune system may enhance viral pathogenesis. ${ }^{19,21}$ The mDCs do not possess high levels of DC-SIGN but they do facilitate Antibody-Dependent Enhancement (ADE) via Fc $\gamma$ IIa and
Fc $\gamma$ IIb receptors. ${ }^{18,22}$ ADE in mDCs can increase viral RNA production over 100-folds making dendritic cells potent components in dengue pathogenesis. ${ }^{3,22}$ When DCs fail to mature properly, they will fail to stimulate T-cells and may induce tolerance. ${ }^{22}$ Moreover, mDCs can also activate B-cells through co-stimulation of CD40, IL-6 and IL-14. In addition to DCs, macrophage, monocyte and natural killer (NK) cells also mediate for viral replication, cytokines production and killing of infected cells. ${ }^{22}$ In fact, IL-15 produced by DCs has comparable activity with IL-2 for the induction of B and NK cell proliferation and differentiation. ${ }^{2123}$

Among CSs, methylprednisolone (MP) usually enhance antigen uptake and prevent DC differentiation and maturation and production of TNF-a, IL-6, and IL-12. But MP does not affect the viability of DC. ${ }^{24-26}$ By these ways, the maturity function of DCs is retarded. This may in turn indirectly suggests, reduce dengue viral replication that was 100 times in mDCs. In addition, MP treated DCs were deficient in their ability to elicit proliferative responses and production of MMPs. ${ }^{24}$ This may contribute to prevent vascular leak in dengue. Furthermore, CSs exert potent suppressive effects on human DCs and thereby inhibit the induction of primary $\mathrm{T}$ and $\mathrm{B}$ cell responses preventing immune dysfunction induced by dengue virus. MP also cause up-regulation of the anti-inflammatory cytokine IL-10, which gets its anti-inflammatory effect by suppressing the production of macrophage inflammatory proteins such as IL-1, IL-6, IL-8, IL-12, TNF, the granulocyte-macrophage colony stimulating factor (GM CSF), MHC class II molecules, B7 and intercellular adhesion molecule-1 (ICAM-1). ${ }^{2728}$ More interestingly, IL10 inhibits TNF-induced NF- $\mathrm{kB}$ activity and acts to diminish Th1 cell activity by suppressing IL-2 and interferon- $\gamma \cdot{ }^{29}$ The anti-inflammatory effects of CSs on leucocytes is partly by inhibition of signaling of NF-kB. Moreover, glucocorticoids (GCs) alter the trafficking and functioning of leucocytes such as neutrophils, eosinophils, mast cells and endothelial cells. $^{30}$

Elevated cyclooxygenase-2 (COX-2) and Prostaglandin E2 (PGE2) expression caused by viral infection have been reported to be associated with viral replication and viral pathogenesis. ${ }^{31,32}$ Administration of a single oral dose $(4,8$, or $16 \mathrm{mg}$ ) of MP was found to cause a dose and time-dependent inhibition of whole-blood COX-2 activity and significantly lower the levels of PGE $2^{32}$. Thus it could be suggested that CSs contribute indirectly to reduce both replication and viral pathogenesis either through DCs or COX-2/PGE 2 .

\subsection{CSs suppress $T$ cells, B cells and antibody in dengue pathology (Fig. 2)}

Another important molecule in dengue pathology is the antibody. The normal interaction of dengue virus with anti-dengue antibody generally leads to neutralization. However, in heterotypic dengue viral infections, the antibodies are non-neutralizing and lead to enhancement. Thus pre-existing antibodies (Ig G and Ig M) are associated with antibody-dependent enhancement and complement activation. ${ }^{33}$ In infants also dengue hemorrhagic fever can occur in primary dengue viral infection that could be due to maternally derived nonneutralizing IgG facilitated antibody-dependent enhancement. ${ }^{34}$ The non-neutralizing levels of dengue virus-reactive IgG were postulated to be a critical risk factor for severe dengue during infancy. Therefore live actuated dengue vaccine is a cause for risk of severe dengue in such infants, even in their primary infection. In addition the disease severity due to a robust immune response in infants with primary infections would be associated with a consequence of higher viral burdens in vivo and an activation phenotype of peripheral-blood NK cells and $\mathrm{CD}^{+}$and $\mathrm{CD} 4{ }^{+} \mathrm{T}$ cells. ${ }^{35}$

Type1 T helper (Th1) cells produce IFN $\gamma, \mathrm{IL}-2$ and the tumour necrosis factor (TNF)-beta, which activate macrophages. ${ }^{36,37}$ By contrast, type $2 \mathrm{~T}$ helper (Th2) cells produce IL-4, IL-5, IL-10 and IL-13, which are mainly responsible for antibody production, eosinophil activation and inhibition of several macrophage functions. ${ }^{37}$ IFN $\gamma$ and TNF $\alpha$ were also strongly associated with dengue disease severity and correlate well with T-cell activation. ${ }^{3}$ IFN $\gamma$ secretion by dengue specific T-cells has 


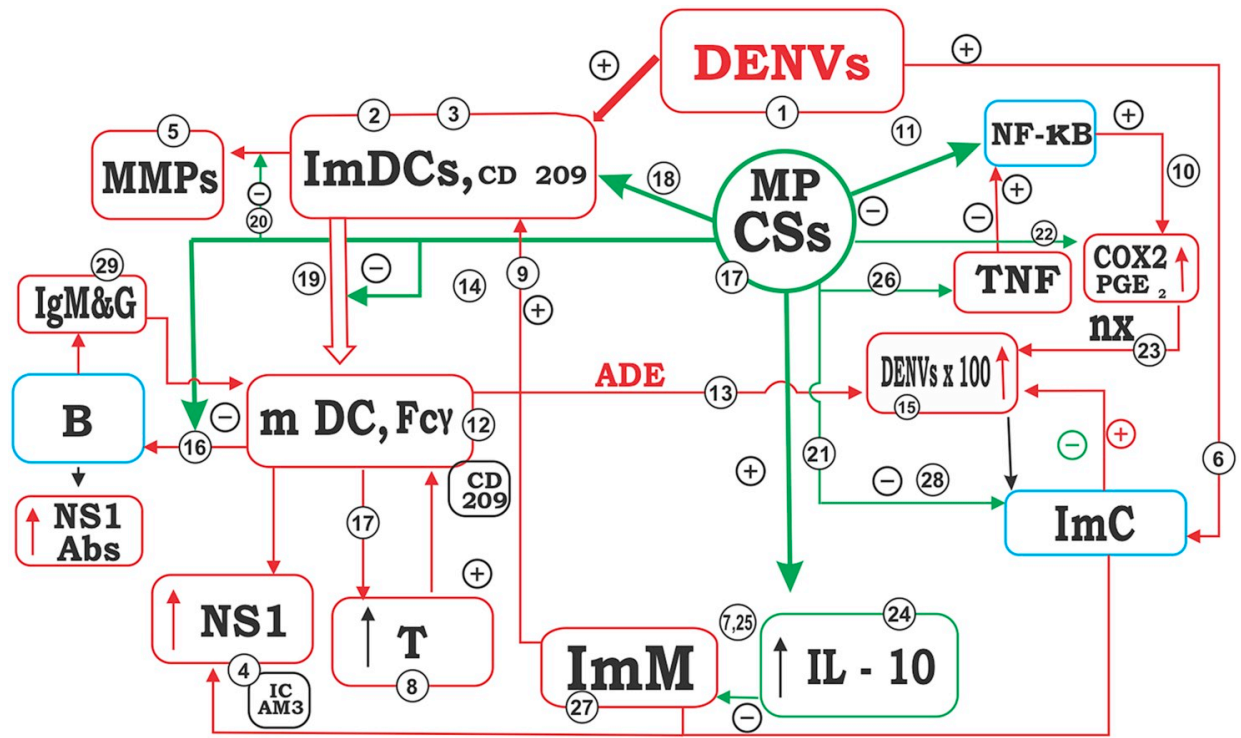

Fig. 1. Steps from dengue virus induce deactivation of dendritic cells and leucocytes to dengue pathogenesis and corticosteroid action in the steps

DENVs (1) infect im DCs (2) is facilitated by CD209(3) which has high affinity to the ICAM3(4)on T cells. MMPs(4) are released by infected DCs and contributes to vascular leakage. DENVs infected immune cells (6) secret cytokines e.g. TNF $\alpha$, IFN $\gamma$, IL-6(7) and activated T cells(8) promote the maturation and sensitivity(9) of imDCs. TNF $\alpha$, IFN $\gamma$ and IL-1B induce COX-2 gene expression through NF kB (10). The antiinflammatory activity of steroids in leukocytes (6) is partly due to inhibition of signaling by NF$\mathrm{kB}(11) . \mathrm{mDCs}(12)$ facilitate $\mathrm{ADE}(13)$ via Fc $\gamma \mathrm{IIa}$ and Fc $\gamma$ IIb receptors (14) that contribute to increase of viral RNA production (15) over 100fold. Macrophages(MQ), monocytes(Mo) and natural killer (NK) cells (6) are also involved in viral replication and cytokines production and killing of infected cells. In addition, mDCs can also activate T and B-cells(16). MP(17) usually

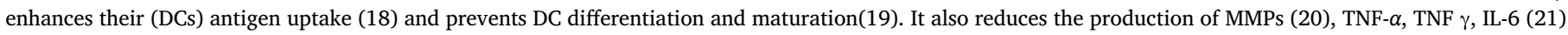

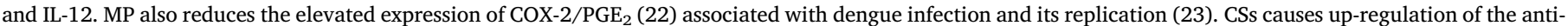

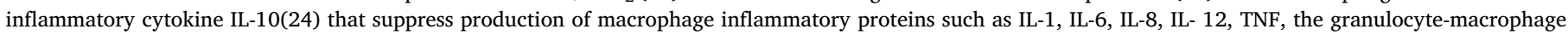

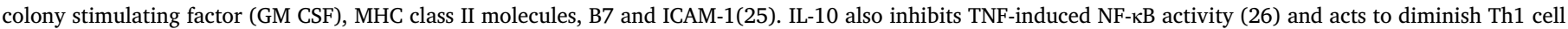

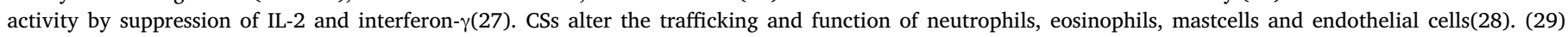
Maternal IgG cause to increase severity of Dengue immune dysfunction in infants.

(DENVs = dengue virus

im DCs = Immature dendritic cells

ICAM = intercellular adhesion molecule

MMPs = producing matrix metalloproteinase

DCs $=$ Dendritic Cells

$\mathrm{TNF} \alpha=$ Tumor necrosis factor alpha

IFN $\gamma=$ Interferon gamma

$\mathrm{IL}=$ Interleukin

COX-2 = cyclooxygenase- 2

$\mathrm{NF} \mathrm{kB}=$ nuclear factor kappaB

mDCs $=$ Mature Dendritic Cells

$\mathrm{ADE}=$ Antibody-Dependent Enhancement

$\mathrm{MP}=$ Methyl prednisolone

$\mathrm{PGE}_{2}=$ cyclooxygenase- 2

Th1 = Type1 $\mathrm{T}$ helper).

been shown to up regulate the number of Fc $\gamma$ receptors, which play a noted role in ADE. ${ }^{38}$ This effect in turn leads to increased replication of dengue virus as well. Interestingly, $\mathrm{CD}^{+}$cells help in controlling early viral infection but the intense proliferation of $\mathrm{CD}^{+}$cells can also contribute to dengue pathogenesis. ${ }^{39}$

CSs inhibit the synthesis of several T cell-derived cytokines at the transcriptional level and perform their action through inhibition of the release of pro-inflammatory cytokines IL- $1 \alpha$, beta, IL-2, IFN $\gamma$, TNF- $\alpha$ and up-regulation of the anti-inflammatory cytokine IL-10 ${ }^{40}$. Another corticosteroid, hydrocortisone had significantly decreased endotoxin induced expression of TNF- $\alpha$, IL-6, IL-8 and IL- 1 and could ameliorate the inflammatory cytokines expression without impairing innate immune responses needed to combat bacterial infections. ${ }^{41}$

More interestingly dengue virus infected cells secrete nonstructural protein 1 (NS1) glycoprotein which can be found bound to platelets, endothelial cells and cells in the lung and liver. ${ }^{42}$ In an in-vitro model of vascular leak, treatment with NS1 alone resulted in the disruption of endothelial cell monolayer integrity ${ }^{43}$ and the vascular leak that plays a major role in the pathology of dengue hemorrhagic fever and shock. Therefore availability of circulating NS1 can be reduced by inhibiting the replication of virus. It could be achieved by early administration of CSs which inhibit whole-blood COX-2 activity and significantly lower the levels of PGE $2^{32}$ reducing both replication of virus and viral pathogenesis either through DCs or COX-2/PGE2.It was also found that no increase of viremia in the early administration of CSs in clinical research. ${ }^{10}$ On the other hand circulating NS1 level also activates toll-like receptor 4 (TLR4) and the TLR2/6 heterodimer in immune cell contributing to the vascular leak that leads to the induction and release of proinflammatory cytokines and chemokines. ${ }^{44}$ On this background it was proposed that NS1 plays a major role in the pathology of dengue hemorrhagic fever and shock via activation of immune cells and NS1induced vascular leak in vitro. Those actions were inhibited by a TLR4 antagonist and by anti-TLR4 antibody treatment ${ }^{44}{ }^{45}$.Moreover, it has also been said that TLR signaling is modulated by even CSs in a cell type-specific fashion resulting in down-regulation of TLR expression, suppression of pro-inflammatory and up-regulation of anti-inflammatory cytokines ${ }^{46}$

On the other hand anti-NS1 antibodies (auto antibody formation) are produced against NS1antigen, leading to the development of an autoimmune pathology in dengue patients. ${ }^{47}$ Moreover, anti-NS1 antibodies show affinity to human fibrinogen, thrombocytes and endothelial cells. ${ }^{42}$ When vulnerable human tissue cells or molecules are exposed to NS1 antibodies, they undergo intrinsic apoptosis, which can be blocked with an inducible nitric oxide synthetase (iNOS) inhibitor. ${ }^{48}$ In addition, anti-NS1 antibodies stimulate the release of IL-6, IL-8 and MCP-1, that involves the activation of complements ${ }^{45}$ in dengue pathology. MP was found to suppress IL-17and IFN- $\gamma$, which are important immune molecules for the autoimmune role in dengue illness induced 


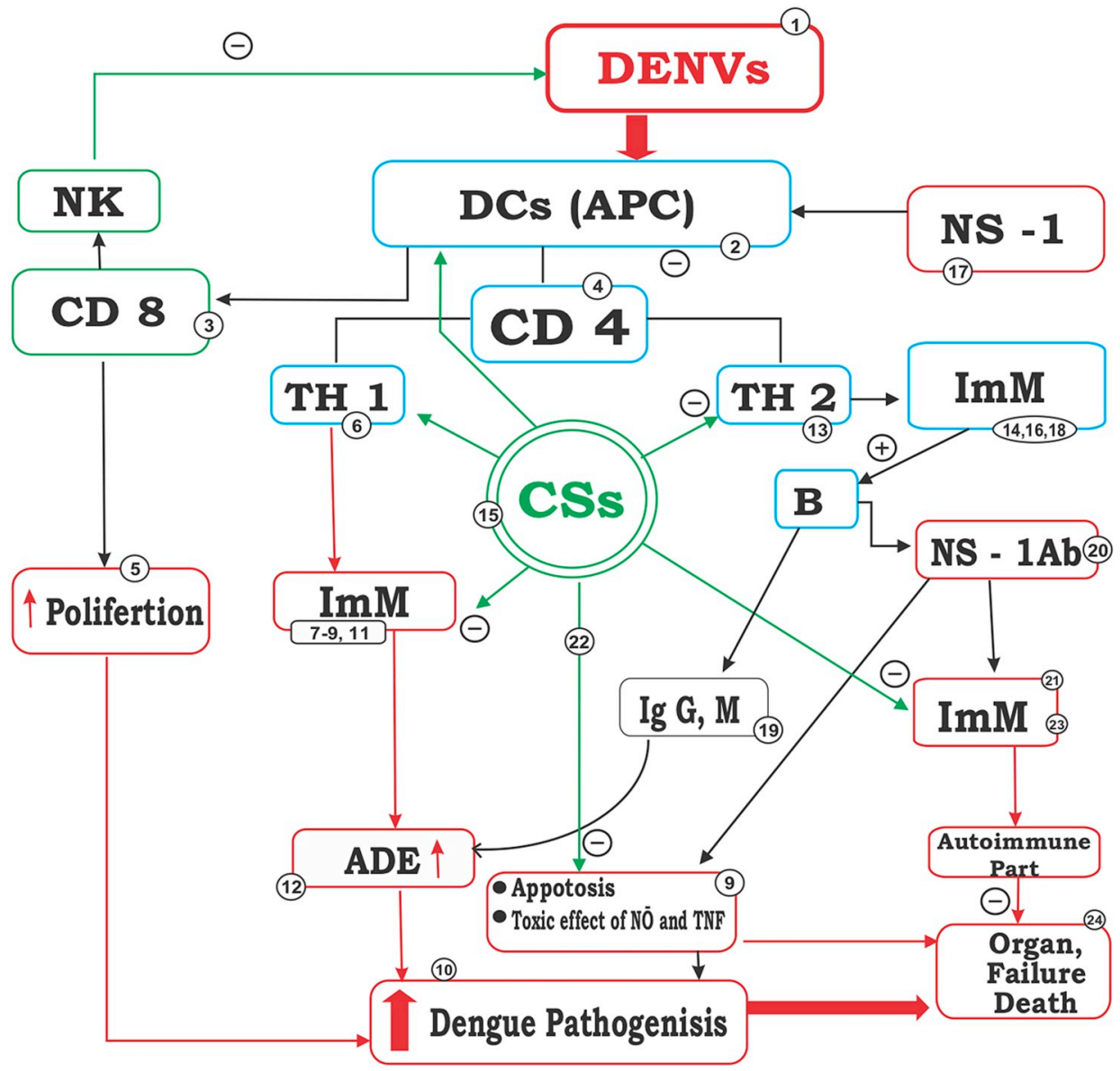

Fig. 2. Steps from activation of $\mathrm{T}$ and $\mathrm{B}$ cells to dengue pathogenesis and corticosteroid action in the steps

DENVs(1) infected DCs or antigen presenting cells(2) activate CD8(3) and CD4 T cells.(4). CD8 + cells mature into NK cells that help in controlling early viral infection (3-1) but the intense proliferation of $\mathrm{CD}^{+}$cells(5) can also contribute to dengue pathogenesis. Activated Type1 T helper (Th1) cells(6) produce interferon-gamma

(IFN $\gamma$ )(7), interleukin (IL)-2, and tumour necrosis factor (TNF)- $\beta$ (8), which activates macrophages (9) to release immune mediators in dengue pathology(10). IFN $\gamma$ up regulates number of $\mathrm{Fc} \gamma$ receptors(11) resulting in increased ADE(12). Activated type 2 Th (Th2) cells (13) produce IL-4, IL-5, IL-10 and IL-13,(14) which are mainly responsible for antibody production, eosinophils activation, and inhibition of several macrophage functions. CSs (15) further involve in the inhibition of the release of proinflammatory cytokines IL-1 $\alpha$ and beta, IL-2, IFN $\gamma$ and TNF- $\alpha$, and up-regulation of the antiinflammatory cytokine IL-10(16). DENVs infected cells secrets NS-1 antigen (17). Antibodies against DENVs and anti-NS1 are produced by B cells which are suppressed by lowering of amounts of IL-2 and IL-2 receptors(18). The former antibodies(19) contribute to $\mathrm{ADE}(12)$ and the latter stimulates immune cells to release of IL-6, IL-8, and MCP-1 and involves in activation of a complement (21). In addition CSs inhibits cytokine-induced apoptosis by up regulating anti-apoptotic genes(22). The autoimmune part of dengue illness is brought about by anti-NS1antibodies mediated via IL-17

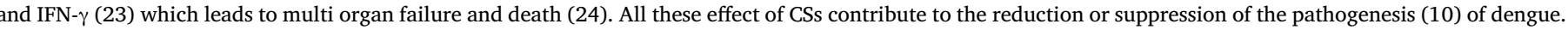
(DENVs $=$ Dengue virus

NK = Natural killer

Th1 = Type1 $\mathrm{T}$ helper

IFN $\gamma=$ interferon-gamma

$\mathrm{TNF}=$ tumour necrosis factor

$\mathrm{IL}=$ Interleukin

CSs $=$ Corticosteroids

$\mathrm{ADE}=$ Antibody-Dependent Enhancement

MCP = Monocyte chemoattractant protein).

by anti NS-1 anti bodies. ${ }^{49}$ Moreover, MP indirectly decreases the cytotoxic effects of nitric oxide (NO) and TNF- $\alpha{ }^{48}$ This effect could help to prevent anti NS1 antibody induced intrinsic apoptosis and vital tissue damage, which may contribute to organ failure and death in dengue. ${ }^{40,50}$ More interestingly, CSs inhibit cytokine-induced apoptosis by up-regulating anti-apoptotic genes and by suppressing humoral immunity through B cells to express lower amounts of IL-2 and IL-2 receptors, ${ }^{40}$ leading to reduce antibody induced immune pathology in dengue patients.

\subsection{Corticosteroids suppress DENVs induced complement activation (Fig. 3)}

The complement system is an important component of the innate immune system against various pathogens. The C3 amplification loop lies at the core of all the complement pathways. ${ }^{51}$ The system controls viral infections through multiple mechanisms, including lysis of virions or infected cells, production of anaphylatoxins and priming of $\mathrm{T}$ and $\mathrm{B}$ cell responses. ${ }^{52}$ However, in dengue infection a deficiency in Mannose- binding lectin (MBL) level or activity due to host polymorphisms in the MBL2 gene correlates with reduced levels of DENV neutralization, which may modulate DHF/DSS manifestations. Therefore, the MBL pathway contributes to protection against DENV infection in humans. ${ }^{5354}$ Moreover, the complement peptides C3a, C4a and C5a are referred to as anaphylatoxins, which have a wide variety of biological functions. ${ }^{51}$ Following dengue viral infections, both DCs and T-cells upregulate C3a and C5a receptors and produce C3 peptide. ${ }^{22}$ Furthermore, the complement peptides are activated by DC antigen uptake and presentation. Some of the primary sources of C3 are APCs such as DC and macrophages. In patients with severe dengue, large amounts of C3a have been detected to serve to recruit monocytes, macrophages and dendritic cells that regulate vasodilatation, increase permeability of small blood vessels, disrupt vasculature and smooth muscle contraction. ${ }^{55}$ These complementary molecules and enzymes can induce oxidative burst and generation of cytotoxic oxygen radicals, mediate chemotaxis, inflammation and basophils, neutrophils, eosinophils and mast cells to release histamine. ${ }^{51} \mathrm{C} 3 \mathrm{a}, \mathrm{C} 5 \mathrm{a}$, and C5b-9 cause circulatory collapse similar to an IgE mediated allergic response e.g. anaphylaxis 


\section{3 - CS s suppress DENVs induced complement activation}

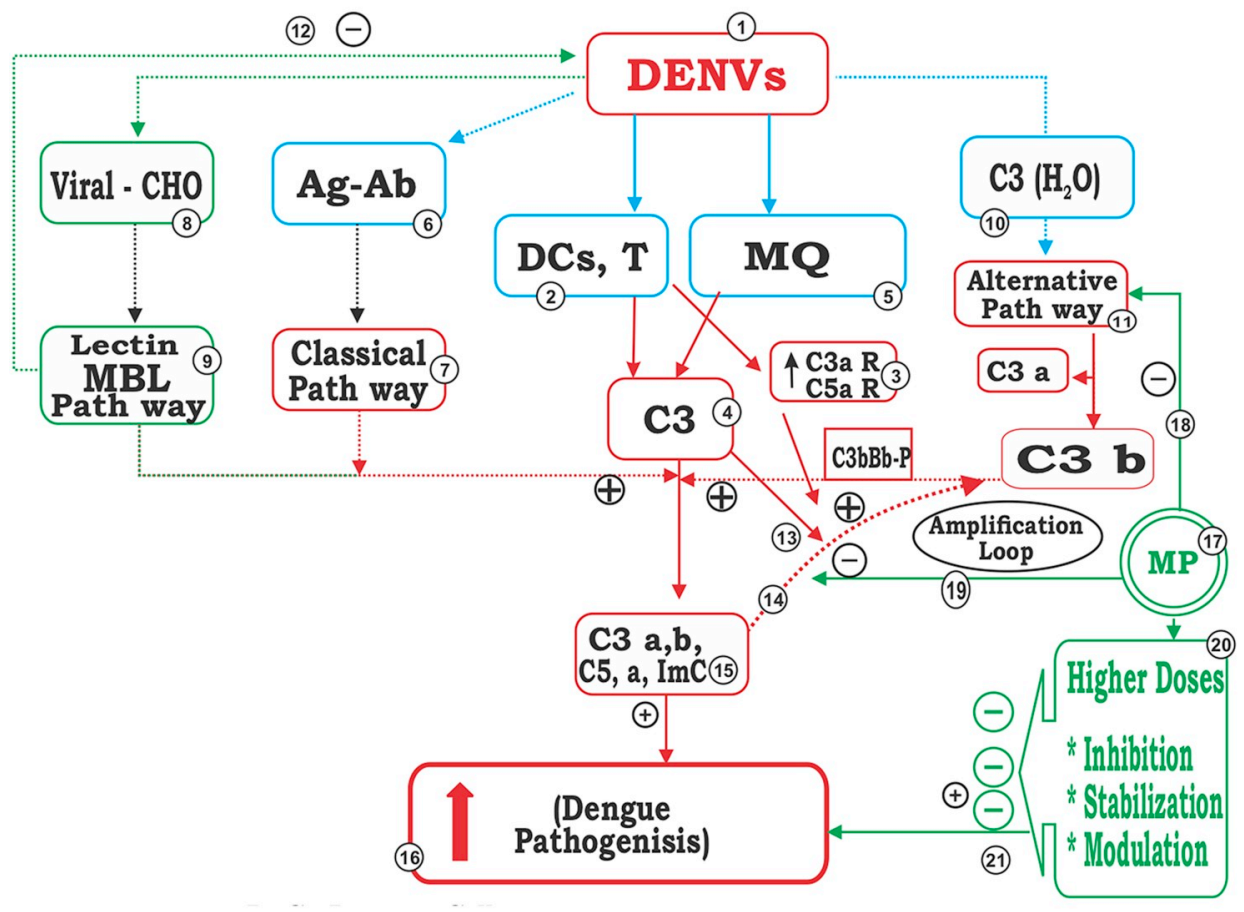

Fig. 3. Steps from complement activation to dengue pathogenesis and action of corticosteroid DENVs(1) infect both DCs and T-cells (2)and upregulate $\mathrm{C} 3 \mathrm{a}$ and $\mathrm{C} 5 \mathrm{a}$ receptors (3) and produce C3 peptide (4).Viral infected macrophage also involve in C3 peptide production (5). The complements are activated through antigen antibody complexes(6) named the classical pathway(7); recognition of carbohydrate structures on pathogens(8) by mannose binding lectin/MBL pathway (9) and hydrolysis of C3(H2O) (10) named as alternative pathway(11). DENVs can be directly neutralized via the MBL pathway (12). The increase in alternative complement proteins, complement receptors(3) and C protein (4), all facilitate (13) a positive feedback loop (amplification pathway)(14). They mediate basophiles, neutrophils, eosinophils and mast cells to release histamine (15). Dengue pathology is increased by complement molecules and enzymes (C3a and C5a) that can induce oxidative bursts, generation of cytotoxic oxygen radicals, chemotaxis, inflammation, circulatory collapse as well (e.g.anaphylaxis)(16). MP(17) directly inhibits the alternative(18) and amplification pathway (19) of the complement but not the MBL path way. CSs in higher doses (20) may function by regulating multiple events in the immunological apparatus including inhibition of compliments, stabilization of membranes and modulation of vivo components levels (21).
$(\mathrm{DENV}=$ Dengue virus

$\mathrm{DC}=$ Dengue virus

MBL $=$ Mannose-binding lectin

MP = Mannose-binding lectin

CSs $=$ Corticosteroids).

when their concentrations are high enough to invoke a general systemic response. ${ }^{56}$ Thus higher complement levels correlate to increasing disease severity of dengue illness. ${ }^{55}$ Cross reactive antibodies activate complements further. Finally increased alternative complement proteins, complement receptors and $\mathrm{C}$ proteins facilitate a positive feedback loop that can lead to dangerous consequences in a dengue-infected patient.

In this life threatening steps of dengue pathology, it is observed that MP directly inhibits the alternative and amplification pathway of complements. ${ }^{57}$ Moreover, steroids in higher doses may regulate multiple steps in the immunological apparatus, including stabilization of membranes, modulation of in vivo component levels and inhibition of complements, which help to stop or prevent histamine release, vascular permeability and circulatory collapse. ${ }^{58}$

\subsection{Corticosteroids suppress DENV induced hematological manifestation (Fig. 4)}

Hematological abnormalities that are generally observed in severe dengue, such as thrombocytopenia, coagulopathy, bleeding and vasculopathy are related to platelet and endothelial dysfunction. ${ }^{59}$ Disseminated intravascular coagulation and major hemorrhages in a minority of patients with severe or prolonged shock may be due to severe thrombocytopenia and the secondary effects of hypoxia and acidosis. ${ }^{59}$ Thrombocytopenia may be due to bone marrow suppression, immune mediated platelet destruction, augmented platelet adhesiveness to vascular endothelial cells and high levels of platelet activating factor (PAF). ${ }^{60}$ Moreover marked thrombocytopenia is evident in patients with elevated IL-1 $\beta$, IL-8, TNF- $\alpha$ and Monocyte chemo-attractant protein-1 (MIP-1). ${ }^{61}$ Spontaneous bleeding is strongly associated with platelet count below 100,000 cells/c.mm or a rapid drop in the platelet count. ${ }^{61}$ In addition, the cause for increased spontaneous bleeding may be brought about by low plasma fibrinogen levels, release of Heparansulphate and secretion of Von Willebrand factor (VWF) in DHF. Low plasma fibrinogen may be due to permeation of fibrinogen through the endothelial cells, development of antibodies potentially cross-reactive to plasminogen and impaired synthesis of fibrinogen. ${ }^{6259}$ Heparansulphate in blood vessels acts like an anti-coagulant and is damaged by the initial cytokine response in DHF and liberated to the circulation. This may be the reason for the prolonged Activated partial thromboplastin time (APTT) in DHF. ${ }^{63}$ Severe dengue is associated with increased circulating levels of VWF, which is associated with thrombocytopenia, clinical bleeding and plasma leakage. ${ }^{64}$ However, the tendency to bleed after a high dose of CSs could not be observed because non-genomic effects of GSs enhance the rapid activation of endothelial nitric oxide synthase (eNOS), which is a possible inhibitor of VWF secretion. ${ }^{65}$

Activation of both platelets and complements and release of inflammatory mediators such as vascular endothelial growth factor (VEGF), TNF $\alpha$, PAF and Monocyte chemoattractant protein-1 (MCP-1) is proposed as the mechanism that causes vasculopathy leading to the plasma leakage. ${ }^{66}$ VEGF may induce permeability via prostanoids and NO or via PAF. ${ }^{67}$ TNF $\alpha$ may lead to permeability via cyclooxygenase induction and the release of prostanoids. ${ }^{68} \mathrm{In}$ fact, it was observed that CSs inhibit the pro angiogenic gene $\mathrm{VEGF}^{69}$ resulting in reduced plasma leakage. PAF is produced and secreted by several types of cells, including mast cells, monocytes, tissue macrophages, platelets, eosinophils, endothelial cells and neutrophil which has also been shown to be involved in the production of many inflammatory cytokines such as $\mathrm{TNF} \alpha$ and IL-1 $\beta .^{70}$ PAF levels were significantly higher in more severe 


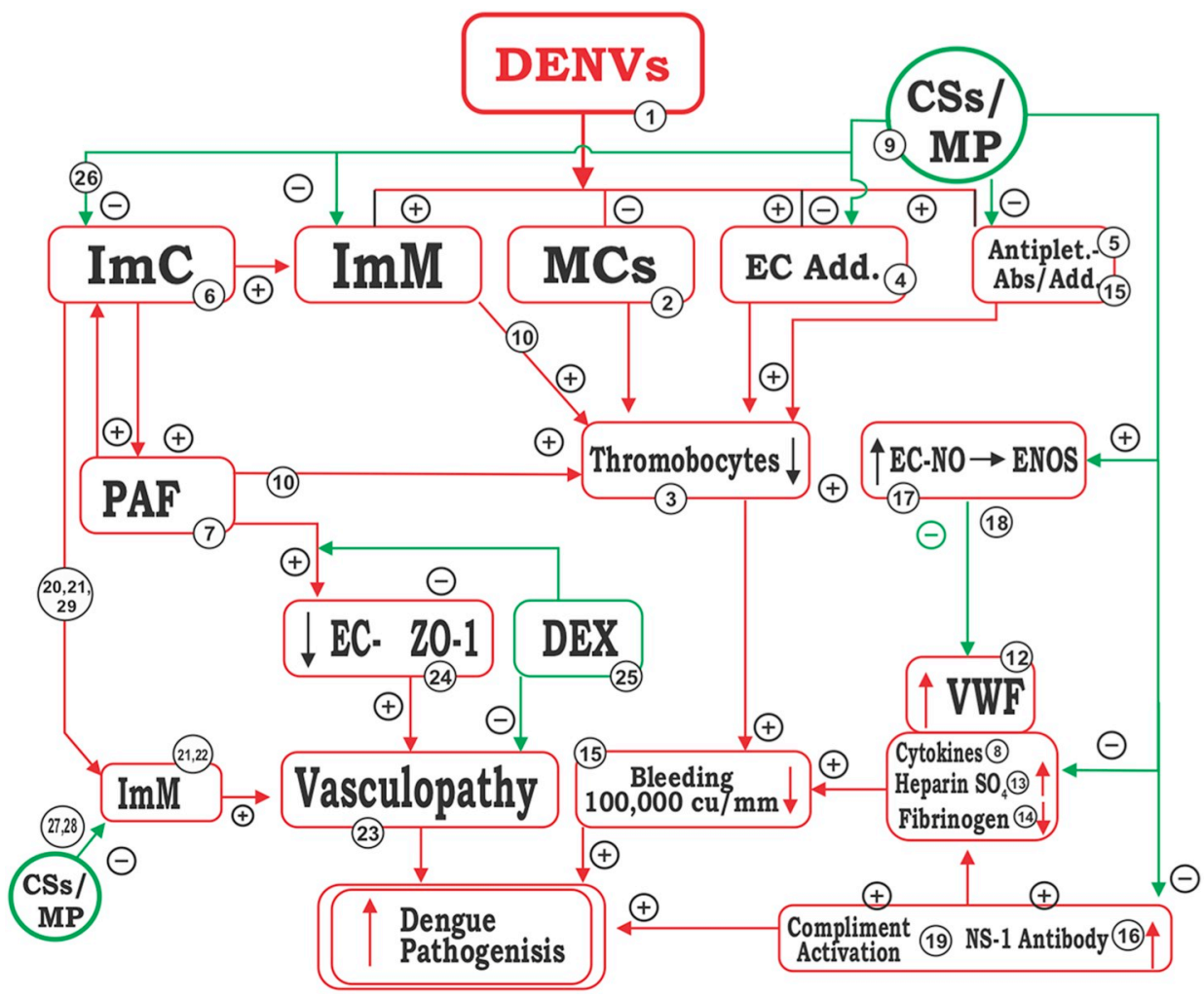

Fig. 4. Steps from hematological manifestation to dengue pathogenesis and corticosteroid action in the steps.

DENVs(1) infect and suppress megakaryocyte cells (MCs) (2) in bone marrow leading to thrombocytopenia(3) that is aggravated by endothelial cells adhesion molecule (4), antiplatelet antibodies(5) and platelet adhesion molecule (15). Infected immune cells(7) secrete platelet activating factor (PAF) (8) and cytokines(9) and chemokines. These effects are suppressed or reduced by CSs (6) PAF is implicated in platelet aggregation and activation (10) resulting thrombocytopenia (3), increased release of VWF (11) and Heparan sulphate(12), low levels of fibrinogen (13) contribute to aggravated bleeding (14) tendency in dengue. The dengue induced pathological effect such as production of cytokines (9), platelet adhesive molecules (15) NS-1 antibody (16) formation are suppressed by CSs/ MP. The ability of increased endothelial NO (e NO)(17) production by MP leads to reduced secretion of (18). All contribute to reduce the tendency to bleed(13) and its cause in dengue. Activation of platelets(10),complements(19) and release of inflammatory mediators such as PAF (8), MCP-1(20), VEGF(21), TNF(22) leading to plasma leakage and contribute to the development of vasculopathy (23). It is brought about by PAF through altered expressions of the pattern of the tight junction protein ZO-1 (24). Dexamethasone (DEX) (25) increases the expression of ZO-1 and decreases levels of PAF via suppression of immune molecules. CSs suppress immune cells(26) and reduce production of prostanoids (PTs), NO (27) and COX-2 (28) which are the permeability facilitators of VEGF and TNF respectively. (29) CSs also suppress the action of NS-1directly and indirectly.

(DENV $=$ Dengue virus

MCs = megakaryocyte cells

$\mathrm{PAF}=$ platelet activating factor

$\mathrm{VWF}=$ Von Willebrand factor

CSs $=$ Corticosteroids

MP = Methylprednisolone

MCP = Monocyte chemoattractant protein

VEGF $=$ Vascular endothelial growth factor

$\mathrm{TNF}=$ Tumor necrosis factor alpha

DEX $=$ Dexamethasone

$\mathrm{PTs}=$ prostanoids

NO $=$ Nitric oxide

COX-2 = Cyclooxygenase-2).

forms of dengue and were associated with a reduced expression of tight junction proteins and reduced cell layer integrity resulting in increased para cellular leak. ${ }^{71}$ Moreover, PAF acts as a potent activator and a mediator of both immune-mediated and non-immune mediated anaphylaxis. It is implicated in platelet aggregation and activation through oxidative bursts, chemotaxis of leukocytes, augmentation of arachidonic acid, metabolism and release of vasoactive amines in the inflammatory response, resulting in increased vascular permeability, circulatory collapse, decreased cardiac output and various other biological effects. ${ }^{70,71}$ In dengue patients, PAF was found to alter the expression pattern of the tight junction protein $\mathrm{ZO}-1$ and had decreased the integrity of human endothelial cell monolayer, as measured by trans-endothelial resistance. ${ }^{71}$ Among the CSs, dexamethasone was investigated earlier and proved that it can increase transendothelial electrical resistance and ZO-1 expression. ${ }^{72}$ In addition, dexamethasone has also been found to influence tight junction expression leading to a reduction of permeability. ${ }^{72}$ Furthermore, it was observed that the amount of plasma extravasations produced by PAF was decreased by dexamethasone in adrenalectomized rats. ${ }^{73}$ On the other hand, TNF and
IL-1were found to stimulate the synthesis and release of the plateletactivating factor (PAF) by neutrophils and vascular endothelial cells, ${ }^{74}$ which could be effectively suppressed by CSs as mentioned above. ${ }^{72}$

\section{Conclusion}

Lack of knowledge and clinical practice on the use of corticosteroids in relation to immune pathology exists and causes clinical manifestations of DSS/DHF and their complications. Therefore all clinicians must be provided with adequate scientific evidence in these regards. Apart from the existing fluid management for dengue disease, this immune pharmacological approach is suggested as a treatment option to suppress the immune dysfunction that leads to DHF and other complication of dengue. Therefore the value of steroid on dengue immune pathology cannot be underestimated and this could open a completely new scientific approach and will be suggested for management of more than $250,000-500,000$ patients in the world who suffer from sever dengue disease annually. This can be tested further with a double blind control trial study using a standard steroid protocol considering the stages of 
immunopathology of dengue. This review article (a) provides an immunological link between dengue and steroid treatment (b) offers scope for corticosteroid based treatment as an approach for DF and DHF,DSS (c) provides a better understanding of actions of corticosteroid in dengue immune pathology (d) provides and opens well known arguments, completely new to dengue, but with strong scientific evidence to reconsider use of steroid to manage dengue and its complications (e) offers scope to consider steroids for primary and secondary prevention of dengue and complication and treatment. (f) provides information on the main cause for aggravation of existing dengue pathology under nonsteroid therapy $(\mathrm{g})$ provides a plausible explanation as to why not all patients with dengue develop DHF/DSS and differently associated complications. (h) can be used to explain other treatment methods that are used to explain such as herbal and other approaches to control dengue disorders and its complications (i) offers a novel coherent picture of the immune pathology of dengue related to the pharmacological approach of corticosteroid treatment at different severity of dengue immune pathology.

\section{Declaration of Competing interest}

None to declare.

\section{Acknowledgements}

I thank all the researchers and their medical and non-medical staff for their dedication to do research on dengue related topics and publishing them for past few decades. I am most grateful to Dr Rohitha Muthugala (Consultant Virologist) Dr. Indunil Wijeweera (Consultant Neurologist Neurology Unit), Dr. R.M.S.K Rathnayake (The Director) and Dr. Nishshanka Wijewardane, (Deputy Director) at General Hospital Kandy. I also thank Mr. P.G.A.C.Siriwardana, Mr.Y.C.Senanayake (fourth year Medical students, university of Peradeniya) Ms.T.N.Senanyake (First year medical student,university of Karapitiya) for their contribution to draw diagrams. I would also like to acknowledge Dr.W.A.W.M.R.M.P.Wijesingha, Dr.Mrs.W.M.J.K.K.Buddadasa, Dr.Mrs.J.Abbas Dr Mrs. Deepa Gunawardana and Dr. Mrs Awanthi (Teaching Hospital Kandy) and the administrative staff at the Teaching Hospital, Kandy. In addition, I extend my gratitude to the Editorial Board of the Journal for taking early steps to publish this.

\section{Abbreviations}

$\begin{array}{ll}\text { DF } & \text { Dengue fever } \\ \text { DHF } & \text { Dengue hemorrhagic fever } \\ \text { DSS } & \text { Dengue shock syndrome } \\ \text { WHO } & \text { World Health Organization } \\ \text { CSs } & \text { Corticosteroids } \\ \text { DENV } & \text { Dengue virus } \\ \text { DCs } & \text { Dendritic cells } \\ \text { imDCs } & \text { Immature dendritic cells } \\ \text { DC-SIGN } & \text { Dendritic Cell Specific Intercellular adhesion molecule-3 } \\ & \text { (ICAM-3) Grabbing Non-integrin } \\ \text { MMPs } & \text { Matrix metalloproteinase } \\ \text { TNFa } & \text { Tumor necrosis factor alpha } \\ \text { IFN } \gamma & \text { Interferon gamma } \\ \text { IL } & \text { Interleukin } \\ \text { mDCs } & \text { Mature Dendritic Cells } \\ \text { MHC } & \text { Major histocompatibility complex } \\ \text { ADE } & \text { Antibody-Dependent Enhancement } \\ \text { NK } & \text { Natural killer } \\ \text { MP } & \text { Methylprednisolone } \\ \text { GM CSF } & \text { Granulocyte-macrophage colony stimulating factor } \\ \text { ICAM-1 } & \text { Intercellular adhesion molecule-1 } \\ \text { GCs } & \text { Glucocorticoids }\end{array}$

COX-2 Cyclooxygenase-2

PGE2 Prostaglandin E2

Th1 Type1 Thelper

Th2 Type $2 \mathrm{Th}$

NS1 Nonstructural protein 1

iNOS Inducible nitric oxide synthatase

NO Nitric oxide

MBL Mannose-binding lectin

PAF Platelet activating factor

VWF Von Willebrand factor

APTT Activated partial thromboplastin time

eNOS Endothelial nitric oxide synthase

VEGF Vascular endothelial growth factor

MCP-1 Monocyte chemoattractant protein-1

\section{Ethical approval}

Not required.

\section{Availability of data and material}

The data set for this publication is available upon request from the authors.

\section{Funding}

None.

\section{Authors contribution}

SMRB Formulated hypotheses on following topics such Corticosteroid actions on dengue immune pathology effectiveness of corticosteroid in dengue treatment and management of dengue and post dengue Syndrome hypothesis for dengue, devised the project and review articles, the main conceptual ideas and proof outline, wrote the manuscript. HMMTBH corrected, edited the manuscript and supervised the review.

\section{References}

1. Bäck AT, Lundkvist Å. Dengue viruses - an overview. Infect Ecol Epidemiol. 2013;3(1):19839. https://doi.org/10.3402/iee.v3i0.19839.

2. Soo K-M, Khalid B, Ching S-M, Chee H-Y. Meta-analysis of dengue severity during infection by different dengue virus serotypes in primary and secondary infections. In: Huy NT, ed. PLoS One. 2016;11(5):e0154760https://doi.org/10.1371/journal.pone. 0154760 .

3. Green S, Rothman A. Immunopathological mechanisms in dengue and dengue hemorrhagic fever. Curr Opin Infect Dis. 2006;19(5):429-436. https://doi.org/10.1097/ 01.qco.0000244047.31135.fa.

4. Hadinegoro SRS. The revised WHO dengue case classification: does the system need to be modified? Paediatr Int Child Health. 2013;32(sup1):33-38. https://doi.org/10. 1179/2046904712Z.00000000052.

5. Jayarajah U, Faizer S, de zoysa I, Seneviratne PS. A large dengue epidemic affects Sri Lanka. 2017 2017;6(1):84-86.

6. Ebi KL, Nealon J. Dengue in a changing climate. Environ Res. 2016;151:115-123. https://doi.org/10.1016/j.envres.2016.07.026.

7. Zhang F, Kramer CV. Corticosteroids for dengue infection. Cochrane infectious diseases group. Cochrane Database Syst Rev. 2014;85(4):525. https://doi.org/10.1002/ 14651858.CD003488.pub3.

8. Bandara SMR, Herath HMMTB. Effectiveness of corticosteroid in the treatment of dengue - a systemic review. Heliyon. 2018;4(9):e00816https://doi.org/10.1016/j. heliyon.2018.e00816.

9. Tam DTH, Ngoc TV, Tien NTH, et al. Effects of short-course oral corticosteroid therapy in early dengue infection in Vietnamese patients: a randomized, placebocontrolled trial. Clin Infect Dis. 2012;55(9):1216-1224. https://doi.org/10.1093/cid/ cis655.

10. Nguyen THT, Nguyen THQ, Vu TT, et al. Corticosteroids for dengue - why don't they work? Halstead SB. PLoS Neglected Trop Dis. 2013;7(12):e2592https://doi.org/10. 1371/journal.pntd.0002592.

11. Tassniyom S, Vasanawathana S, Chirawatkul A, Rojanasuphot S. Failure of high-dose methylprednisolone in established dengue shock syndrome: a placebo-controlled, 
double-blind study. Pediatrics. 1993;92(1):111-115.

12. Premaratna R, Jayasinghe KGNU, Liyanaarachchi EW, Weerasinghe OMS, Pathmeswaran A, de Silva HJ. Effect of a single dose of methyl prednisolone as rescue medication for patients who develop hypotensive dengue shock syndrome during the febrile phase: a retrospective observational study. Int $J$ Infect Dis. 2011;15(6):e433-e434. https://doi.org/10.1016/j.ijid.2011.03.006.

13. Flores AYL, Tapia MP, Garcia IE, et al. Dengue virus inoculation to human skin explants: an effective approach to assess in situ the early infection and the effects on cutaneous dendritic cells. Int $J$ Exp Pathol. 2005;86(5):323-334. https://doi.org/10. 1111/j.0959-9673.2005.00445.x.

14. Durbin AP, Vargas MJ, Wanionek K, et al. Phenotyping of peripheral blood mononuclear cells during acute dengue illness demonstrates infection and increased activation of monocytes in severe cases compared to classic dengue fever. Virology. 2008;376(2):429-435. https://doi.org/10.1016/j.virol.2008.03.028.

15. Blackley S, Kou Z, Chen H, et al. Primary human splenic macrophages, but not T or B cells, are the principal target cells for dengue virus infection in vitro. $J$ Virol. 2007;81(24):13325-13334. https://doi.org/10.1128/JVI.01568-07.

16. Lozach P-Y, Burleigh L, Staropoli I, et al. Dendritic cell-specific intercellular adhesion molecule 3-grabbing non-integrin (DC-SIGN)-mediated enhancement of dengue virus infection is independent of DC-SIGN internalization signals. $J$ Biol Chem. 2005;280(25):23698-23708. https://doi.org/10.1074/jbc.M504337200.

17. Boonnak K, Slike BM, Burgess TH, et al. Role of dendritic cells in antibody-dependent enhancement of dengue virus infection. $J$ Virol. 2008;82(8):3939-3951. https://doi. org/10.1128/JVI.02484-07.

18. Pokidysheva E, Zhang Y, Battisti AJ, et al. Cryo-EM reconstruction of dengue virus in complex with the carbohydrate recognition domain of DC-SIGN. Cell. 2006;124(3):485-493. https://doi.org/10.1016/j.cell.2005.11.042.

19. Tirado SMC, Yoon K-J. Antibody-dependent enhancement of virus infection and disease. Viral Immunol. 2003;16(1):69-86. https://doi.org/10.1089/ 088282403763635465

20. Palmer DR, Sun P, Celluzzi C, et al. Differential effects of dengue virus on infected and bystander dendritic cells. $J$ Virol. 2005;79(4):2432-2439. https://doi.org/10. 1128/JVI.79.4.2432-2439.2005.

21. Henrickson SE, Mempel TR, Mazo IB, et al. T cell sensing of antigen dose governs interactive behavior with dendritic cells and sets a threshold for T cell activation. Nat Immunol. 2008;9(3):282-291. https://doi.org/10.1038/ni1559.

22. Nielsen DG. The relationship of interacting immunological components in dengue pathogenesis. Virol J. 2009;6(1):211. https://doi.org/10.1186/1743-422X-6-211.

23. Armitage RJ, Macduff BM, Eisenman J, Paxton R, Grabstein KH. IL-15 has stimulatory activity for the induction of B cell proliferation and differentiation. $J$ Immuno 1995;154(2):483-490.

24. Vanderheyde N, Verhasselt V, Goldman M, Willems F. Inhibition of human dendritic cell functions by methylprednisolone. Transplantation. 67(10):1342.

25. Piemonti L, Monti P, Allavena P, et al. Glucocorticoids affect human dendritic cell differentiation and maturation. J Immunol. 1999;162(11):6473-6481.

26. Wong P, Cuello C, Bertouch JV, et al. The effects of pulse methylprednisolone on matrix metalloproteinase and tissue inhibitor of metalloproteinase-1 expression in rheumatoid arthritis. Rheumatology. 2000;39(10):1067-1073. https://doi.org/10. 1093/rheumatology/39.10.1067.

27. Moore KW, de Waal Malefyt R, Coffman RL, O'Garra A. Interleukin-10 and the in terleukin-10 receptor. Ann. Rev. 2003;19(1):683-765. https://doi.org/10.1146/ annurev.immunol.19.1.683.

28. Hodge Flower. Han. Methyl-prednisolone up-regulates monocyte interleukin-10 production in stimulated whole blood. Scand J Immunol. 1999;49(5):548-553. https://doi.org/10.1046/j.1365-3083.1999.00538.x.

29. Coutinho AE, Chapman KE. The anti-inflammatory and immunosuppressive effects of glucocorticoids, recent developments and mechanistic insights. Mol Cell Endocrinol. 2011;335(1):2-13. https://doi.org/10.1016/j.mce.2010.04.005.

30. Santini G. The human pharmacology of monocyte cyclooxygenase 2 inhibition by cortisol and synthetic glucocorticoids. Clin Pharmacol Ther. 2001;70(5):475-483. https://doi.org/10.1067/mcp.2001.119213.

31. Steer SA, Corbett JA. The role and regulation of COX-2 during viral infection. Viral Immunol. 2003;16(4):447-460. https://doi.org/10.1089/088282403771926283.

32. Lin C-K, Tseng C-K, Wu Y-H, et al. Cyclooxygenase-2 facilitates dengue virus replication and serves as a potential target for developing antiviral agents. Sci Rep. 2017;7(1):299. https://doi.org/10.1038/srep44701.

33. Tirado SMC, Yoon K-J. Antibody-dependent enhancement of virus infection and disease. Viral Immunol. 2004;16(1):69-86. https://doi.org/10.1089/ 088282403763635465 .

34. Chau TNB, Hieu NT, Anders KL, et al. Dengue virus infections and maternal antibody decay in a prospective birth cohort study of Vietnamese infants. $J$ Infect Dis. 2009;200(12):1893-1900. https://doi.org/10.1086/648407.

35. Chau TNB, Quyen NTH, Thuy TT, et al. Dengue in Vietnamese infants-results of infection-enhancement assays correlate with age-related disease epidemiology, and cellular immune responses correlate with disease severity. $J$ Infect Dis. 2008;198(4):516-524. https://doi.org/10.1086/590117.

36. Swain SL, McKinstry KK, Strutt TM. Expanding roles for CD4 + T cells in immunity to viruses. Nat Rev Immunol. 2012;12(2):136-148. https://doi.org/10.1038/nri3152.

37. Weiskopf D, Sette A. T-cell immunity to infection with dengue virus in humans. Front Immunol. 2014;5(Pt 1):571. https://doi.org/10.3389/fimmu.2014.00093.

38. Kontny U, Kurane I, Ennis FA. Gamma interferon augments Fc gamma receptormediated dengue virus infection of human monocytic cells. $J$ Virol. 1988;62(11):3928-3933.
39. de Matos AM, Carvalho KI, Rosa DS, et al. CD8 + T lymphocyte expansion, proliferation and activation in dengue fever. In: Harris E, ed. PLOS Neglected Tropical Diseases. vol. 9. 2015; 2015e0003520https://doi.org/10.1371/journal.pntd. 00035202.

40. Coutinho AE, Chapman KE. The anti-inflammatory and immunosuppressive effects of glucocorticoids, recent developments and mechanistic insights. Mol Cell Endocrinol. 2011;335(1):2-13. https://doi.org/10.1016/j.mce.2010.04.005.

41. Hart KA, Barton MH, Vandenplas ML, Hurley DJ. Effects of low-dose hydrocortisone therapy on immune function in neonatal horses. Pediatr Res. 2011;70(1):72-77. https://doi.org/10.1203/PDR.0b013e31821b502b 1971 5:7.

42. Avirutnan P, Zhang L, Punyadee N, et al. Secreted NS1 of dengue virus attaches to the surface of cells via interactions with heparan sulfate and chondroitin sulfate E. PLoS Pathog. 2007;3(11):e183. https://doi.org/10.1371/journal.ppat.0030183.

43. Modhiran N, Watterson D, Muller DA, et al. Dengue virus NS1 protein activates cells via Toll-like receptor 4 and disrupts endothelial cell monolayer integrity. Sci Transl Med. 2015;7(304) https://doi.org/10.1126/scitranslmed.aaa3863 304ra142$304 \mathrm{ra} 142$.

44. Modhiran N, Watterson D, Blumenthal A, Baxter AG, Young PR, Stacey KJ. Dengue virus NS1 protein activates immune cells via TLR4 but not TLR2 or TLR6. Immunol Cell Biol. 2017;95(5):491-495. https://doi.org/10.1038/icb.2017.5.

45. Avirutnan P, Punyadee N, Noisakran S, et al. Vascular leakage in severe dengue virus infections: a potential role for the nonstructural viral protein NS1 and complement. $J$ Infect Dis. 2006;193(8):1078-1088. https://doi.org/10.1086/500949.

46. Broering R, Montag M, Jiang M, et al. Corticosteroids shift the Toll-like receptor response pattern of primary-isolated murine liver cells from an inflammatory to an anti-inflammatory state. Int Immunol. 2011;23(9):537-544. https://doi.org/10. 1093/intimm/dxr048.

47. Lin C-F, Wan S-W, Cheng H-J, Lei H-Y, Lin Y-S. Autoimmune pathogenesis in dengue virus infection. Viral Immunol. 2006;19(2):127-132. https://doi.org/10.1089/vim. 2006.19.127.

48. Lin CF, Lei HY, Shiau AL, et al. Endothelial cell apoptosis induced by antibodies against dengue virus nonstructural protein 1 via production of nitric oxide. $J$ Immunol. 2002;169(4) https://doi.org/10.4049/jimmunol.169.4.2215 2215-2215.

49. Elsabahy M, Wooley KL. Cytokines as biomarkers of nanoparticle immunotoxicity. Chem Soc Rev. 2013;42(12):5552. https://doi.org/10.1039/c3cs60064e.

50. Meßmer UK, Winkel G, Briner VA, Pfeilschifter J. Suppression of apoptosis by glucocorticoids in glomerular endothelial cells: effects on proapoptotic pathways. $\mathrm{Br} J$ Pharmacol. 2000;129(8):1673-1683. https://doi.org/10.1038/sj.bjp.0703255.

51. Harboe M, Mollnes TE. The alternative complement pathway revisited. $J$ Cell Mol Med. 2008;12(4):1074-1084. https://doi.org/10.1111/j.1582-4934.2008.00350.x.

52. Shresta S. Role of complement in dengue virus infection: protection or pathogenesis? mBio. 2012;3(1):476. https://doi.org/10.1128/mBio.00003-12.

53. Madsen HO, Garred P, Kurtzhals JAL, et al. A new frequent allele is the missing link in the structural polymorphism of the human mannan-binding protein. Immunogenetics. 1994;40(1):37-44. https://doi.org/10.1007/BF00163962.

54. Basta M. Ambivalent effect of immunoglobulins on the complement system: activation versus inhibition. Mol Immunol. 2008;45(16):4073-4079. https://doi.org/10. 1016/j.molimm.2008.07.012.

55. Yamanaka A, Hendrianto E, Mulyatno KC, et al. Correlation between complement component levels and disease severity in dengue patients in Indonesia. Jpn J Infect Dis. 2013;66(5):366-374. https://doi.org/10.7883/yoken.66.366.

56. He S-H, Zhang H-Y, Zeng X-N, Chen D, Yang P-C. Mast cells and basophils are essential for allergies: mechanisms of allergic inflammation and a proposed procedure for diagnosis. Acta Pharmacol Sin. 2013;34(10):1270-1283. https://doi.org/10. 1038/aps.2013.88.

57. Weiler JM, Packard BD. Methylprednisolone inhibits the alternative and amplification pathways of complement. Infect Immun. 1982;38(1):122-126.

58. Hammerschmidt DE, White JG, Craddock PR, Jacob HS. Corticosteroids inhibit complement-induced granulocyte aggregation. A possible mechanism for their efficacy in shock states. J Clin Investig. 1979;63(4):798-803. https://doi.org/10.1172/ JCI109365.

59. Azeredo EL de, Monteiro RQ, de-Oliveira Pinto LM. Thrombocytopenia in dengue: interrelationship between virus and the imbalance between coagulation and fibrinolysis and inflammatory mediators. Mediat Inflamm. 2015;2015(3):1-16. https:// doi.org/10.1155/2015/313842.

60. Wang S, He R, Patarapotikul J, Innis BL, Anderson R. Antibody-enhanced binding of dengue-2 virus to human platelets. Virology. 1995;213(1):254-257. https://doi.org/ 10.1006/viro.1995.1567.

61. Bozza FA, Cruz OG, Zagne SMO, et al. Multiplex cytokine profile from dengue patients: MIP-1beta and IFN-gamma as predictive factors for severity. BMC Infect Dis. 2008;8(1):86. https://doi.org/10.1186/1471-2334-8-86.

62. Wills BA, Oragui EE, Minh Dung N, et al. Size and charge characteristics of the protein leak in dengue shock syndrome. J Infect Dis. 2004;190(4):810-818. https:// doi.org/10.1086/422754.

63. Palmer RN, Rick ME, Rick PD, Zeller JA, Gralnick HR. Circulating heparan sulfate anticoagulant in a patient with a fatal bleeding disorder. $N$ Engl $J$ Med. 1984;310(26):1696-1699. https://doi.org/10.1056/NEJM198406283102603.

64. Nehmé A, Edelman J. Dexamethasone inhibits high glucose-, TNF-alpha-, and IL1beta-induced secretion of inflammatory and angiogenic mediators from retinal microvascular pericytes. Investig Ophthalmol Vis Sci. 2008;49(5):2030-2038. https:// doi.org/10.1167/iovs.07-0273.

65. Chopra A, Kumar R, Kishore K, et al. Effect of glucocorticoids on von Willebrand factor levels and its correlation with von Willebrand factor gene promoter 
polymorphism. Blood Coagul Fibrinolysis. 2012;23(6):514-519. https://doi.org/10. 1097/MBC.0b013e3283548dfc.

66. Mukerjee R, Chaturvedi UC, Dhawan R. Dengue virus-induced human cytotoxic factor: production by peripheral blood leucocytes in vitro. Clin Exp Immunol. 1995;102(2):262-267.

67. Murohara T, Horowitz JR, Silver M, et al. Vascular endothelial growth factor/vascular permeability factor enhances vascular permeability via nitric oxide and prostacyclin. Circulation. 1998;97(1):99-107. https://doi.org/10.1161/01.CIR.97.1.99.

68. Cirino G. Multiple controls in inflammation: extracellular and intracellular phospholipase A2, inducible and constitutive cyclooxygenase, and inducible nitric oxide synthase. Biochem Pharmacol. 1998;55(2):105-111. https://doi.org/10.1016/S00062952(97)00215-3.

69. Gille J, Reisinger K, Westphal-Varghese B, Kaufmann R. Decreased mRNA stability as a mechanism of glucocorticoid-mediated inhibition of vascular endothelial growth factor gene expression by cultured keratinocytes. J Investig Dermatol. 2001:117(6):1581-1587. https://doi.org/10.1046/j.0022-202x.2001.01573.x.

70. Valone FH, Epstein LB. Biphasic platelet-activating factor synthesis by human monocytes stimulated with IL-1-beta, tumor necrosis factor, or IFN-gamma. $J$ Immunol. 1988;141(11):3945-3950.

71. Yang KD, Lee CS, Shaio MF. A higher production of platelet activating factor in ex vivo heterologously secondary dengue-2 virus infections. Acta Microbiol Immunol Hung. 1995;42(4):403-407.

72. Hue CD, Cho FS, Cao S, Dale Bass CR, Meaney DF, Morrison B. Dexamethasone potentiates in vitro blood-brain barrier recovery after primary blast injury by glucocorticoid receptor-mediated upregulation of ZO-1 tight junction protein. $J$ Cereb Blood Flow Metab. 2015;35(7):1191-1198. https://doi.org/10.1038/jcbfm.2015.38.

73. Boschetto $\mathrm{P}$, Musajo FG, Tognetto L, et al. Increase in vascular permeability produced in rat airways by PAF: potentiation by adrenalectomy. $\mathrm{Br} J$ Pharmacol. 1992;105(2):388-392. https://doi.org/10.1111/(ISSN)1476-5381.

74. Aikawa T, Hirose T, Matsumoto I, et al. Effect of platelet-activating factor on cortisol and corticosterone secretion by perfused dog adrenal. Platelet-Activating Factor and Structurally Related Alkyl Ehter Lipids. vol. 101. AOCS Publishing; 2009:1108-1111. https://doi.org/10.1201/9781439832042.ch27. 